\title{
Estimação de parâmetros genéticos para produção de leite de cabras da raça Alpina
}

\author{
Fernanda Cristina Breda1, Lucia Galvão Albuquerque², Marcos Yamaki ${ }^{3}$, João Cruz Reis Filho', \\ José Lindenberg Rocha Sarmento ${ }^{1}$, Paulo Sávio Lopes ${ }^{4}$, Marcelo Teixeira Rodrigues ${ }^{4}$ \\ 1 Pós-Graduação em Genética e Melhoramento Animal - UFV. \\ 2 UNESP. \\ ${ }^{3}$ Pós-Graduação em Zootecnia - UFV. \\ ${ }^{4}$ Departamento de Zootecnia - UFV.
}

RESUMO - Foram utilizados 9.374 registros semanais de produção de leite de 302 primeiras lactações de cabras da raça Alpina. A produção de leite no dia do controle foi analisada por meio de um modelo animal, unicarater, de regressão aleatória, em que as funções de covariâncias para os componentes genéticos aditivos e de ambiente permanente foram modeladas por meio das funções de Wilmink, Ali e Schaeffer e por polinômios ortogonais, em uma escala de Legendre de ordens cúbica e quíntica. Assumiuse, ainda, variância residual homogênea durante toda a lactação e heterogênea com três e quatro classes de variância residual. Os modelos foram comparados pelo critério de informação de Akaike (AIC), pelo critério de informação Bayesiano de Schwar (BIC), pela função de verossimilhança ( $\mathrm{Ln}$ L), pela visualização das estimativas de variâncias genéticas, de ambiente permanente, fenotípicas e residuais e pelas herdabilidades. O polinômio de Legendre de ordem quíntica, com quatro e três classes de variâncias residuais, e a função de Ali e Schaeffer, com quatro classes de variâncias residuais, foram indicados como os mais adequados pelo AIC, BIC e Ln L. Estes modelos diferiram na partição da variância fenotípica para as variâncias de ambiente permanente, genética e residual apenas no início e no final da lactação. Contudo, a função de Ali e Schaeffer resultou em estimativas negativas de correlação genética entre os controles mais distantes. O polinômio de Legendre de ordem quíntica, assumindo variância residual heterogênea, mostrou-se mais adequado para ajustar a produção de leite no dia do controle de cabras da raça Alpina.

Palavras-chave: função de Ali e Schaeffer, função de Wilmink, polinômio ortogonal de Legendre, regressão aleatória

\section{Genetic parameters estimation for test day milk yield of Alpina goats}

ABSTRACT - Data consisting of 9,374 test day milk yield records from 302 first lactations of Alpina goats were analyzed by random regression models using the Wilmink and Ali and Schaeffer functions and Legendre orthogonal polynomials of third and fifth orders. Models including animal additive genetic, permanent environmental and homogeneous or heterogeneous (three or four classes) residual random effects were compared by Akaike information criterion (AIC), Schwarz Bayesian information criterion (BIC), likelihood ratio test (Ln L), phenotypic, permanent environmental, genetic and residual variances and by heritability estimates. According to AIC, BIC and Ln L, Legendre orthogonal polynomial of fifth order with three or four residual classes and Ali and Schaeffer function with four residual classes were the best fitting models. These models differed by the partition of phenotypic, permanent environmental, genetic and residual variance estimates in the beginning and in the end of the lactation period. Genetic correlation estimates between milk yields in the beginning and in the end of lactation obtained by Ali and Schaeffer function were negative. Legendre polynomial of fifth order assuming heterogeneous residual variance was the best fitting model for test day milk yield of Alpina goats.

Key Words: Ali and Schaeffer function, Legendre orthogonal polynomial, random regression, Wilmink function

\section{Introdução}

O crescente desenvolvimento da caprinocultura nos últimos anos no Brasil tem exigido maior acompanhamento pelos técnicos responsáveis pelo melhoramento dos rebanhos. Essa atividade tem se consolidado por sua importância na produção de um alimento de rico valor nutricional, com atrativo preço para o produtor (Gonçalves et al., 2001) e por consistir-se em fonte de renda para populações rurais de média e baixa renda (Lôbo et al., 2003).

No entanto, existem poucos estudos em melhoramento genético envolvendo a produção de leite das raças caprinas e seus mestiços, principalmente em razão do alto custo do controle leiteiro nesses animais. A maioria dos trabalhos 
com cabras leiteiras tem-se restringindo ao estudo da produção total (Soares Filho et al., 2001; Gonçalves et al., 2002; Pimenta Filho et al., 2004)e, mais recentemente, ao emprego de modelos de produção no dia de controle, os chamados modelos de test-day (Sullivan \& Wiggans, 2000; Breznik etal., 2000).

A utilização de metodologias que possam estimar os parâmetros genéticos e predizer os valores genéticos dos animais de forma mais acurada pode contribuir para o incremento dos ganhos obtidos com a seleção. Neste contexto, de acordo com Meyer (2003), os modelos de regressão aleatória têm sido indicados para análises de medidas repetidas ao longo de uma escala contínua, por possibiliar a predição do valor genético dos animais em lactação, mesmo utilizando controles leiteiros de lactações incompletas, viabilizando o uso de maior número de registros da produção nas avaliações dos animais (Freitas, 2003). Além disso, possibilitam a correção para efeitos de ambiente específicos para o dia do controle, permitem a estimação de componentes de (co)variâncias e a predição de valores genéticos em qualquer ponto da curva de lactação, no intervalo em que as medidas foram tomadas.

Polinômios ortogonais e funções paramétricas, como as de Wilmink (Wilmink, 1987) e a de Ali e Schaeffer (Ali \& Schaeffer, 1987), são aplicados na modelagem de dados longitudinais, como curvas de lactação para vacas de leite (Jamrozik et al., 1997; Pool \& Mewissen, 1999; Brotherstone et al., 2000; Jakobsen et al., 2002; Freitas, 2003). Modelos de regressão aleatória também têm sido aplicados em cabras leiteiras, utilizando-se a mesma estrutura de produção no dia do controle. As diferenças estariam na definição de subclasses para as curvas fixas, de intervalos de tempo e de ordem das regressões que deverão ser usadas (Schaeffer, 2004). Contudo, estudos desta natureza em caprinos leiteiros são escassos no Brasil, destacando-se a pesquisa conduzida por Sarmento et al. (2003).
Assim, objetivou-se estimar os componentes de (co)variâncias e parâmetros genéticos para a produção de leite de cabras da raça Alpina, utilizando-se as funções paramétricas e os polinômios ortogonais de Legendre em modelos de regressão aleatória.

\section{Material e Métodos}

Utilizaram-se 9.374 registros semanais de produção de leite de 302 primeiras lactações (encerradas ou incompletas) de cabras alpinas, pertencentes ao Setor de Caprinocultura da Universidade Federal de Viçosa. Os controles foram realizados por ordenha mecânica, duas vezes ao dia. Os animais foram mantidos em baias coletivas sob o sistema de estabulação livre com alimentação à base de silagem de milho, feno e mistura concentrada.

Os dados analisados foram coletados entre os anos de 1997 e 2004. Foram considerados os registros de controles da 2a a 39a semana de lactação (270 dias), sendo eliminadas lactações com menos de seis controles, produções em grupos contemporâneos (rebanho-ano-quinzena do controle) com menos de cinco cabras e produções superiores ou inferiores a três desvios-padrão da média da semana de lactação. $\mathrm{O}$ número de observações, a média e o desvio-padrão por semana podem ser visualizados na Figura 1.

A produção de leite no dia do controle foi analisada por meio de um modelo animal, unicarater, de regressão aleatória, considerando efeitos fixos o grupo contemporâneo (138 níveis) e efeitos linear e quadrático da covariável a idade da cabra ao parto, em meses. Em todos os modelos, a trajetória média da população foi modelada por polinômios ortogonais de Legendre de ordem cúbica.

Na forma matricial, o modelo é descrito como:

$$
y=X b+Z a+W a p+e,
$$

em que y é o vetor das $\mathrm{N}$ observações, medidas em $\mathrm{Nd}$

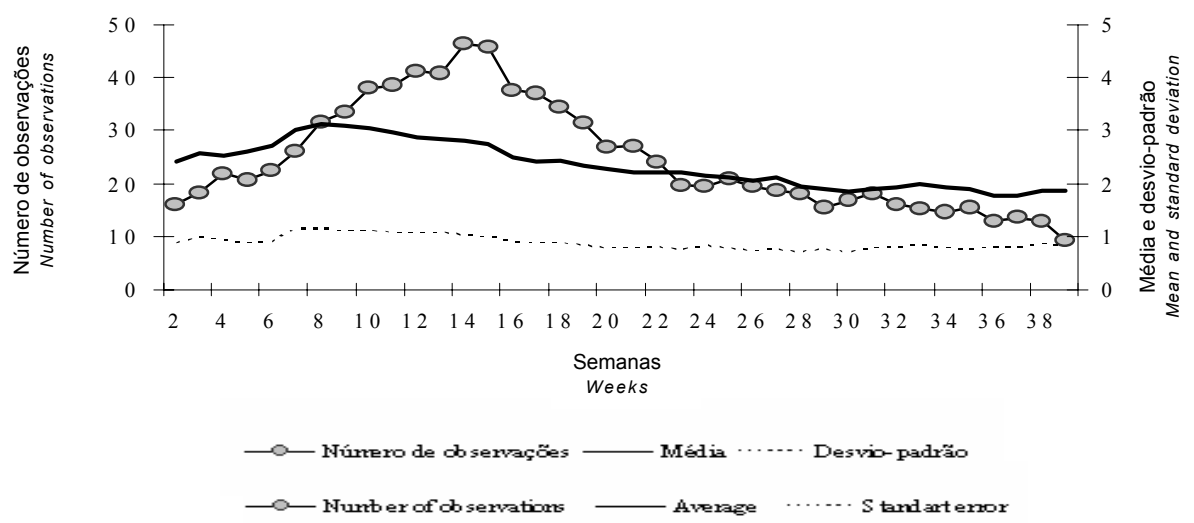

Figura 1 - Número de observações (dividido por 10), produção de leite média e desvio-padrão por semana. Figure 1 - Number of observations (divided by 10), milk yield means and standard deviation per week. 
animais; b, o vetor de efeitos fixos que inclui as soluções para o grupo contemporâneo e para as covariáveis, idade ao parto e semana de lactação; a e ap, os vetores dos coeficientes de regressão aleatórios genético-aditivos e de ambiente permanente, respectivamente; e, o vetor dos diferentes resíduos; e X, Z e W, as matrizes de incidência para os efeitos fixos e aleatórios genéticos aditivos e de ambiente permanente, respectivamente.

A dimensão do vetor a é de $\mathrm{k}_{\mathrm{a}} \times \mathrm{N}_{\mathrm{a}}$ coeficientes, sendo $\mathrm{k}_{\mathrm{a}}$ a ordem da função de regressão e $\mathrm{N}_{\mathrm{a}}$ o número de animais na matriz de parentesco. $\mathrm{O}$ vetor ap tem dimensão de $\mathrm{k}_{\mathrm{ap}} \times \mathrm{N}_{\mathrm{d}}$ coeficientes, sendo $\mathrm{k}_{\mathrm{ap}}$ a ordem da função de regressão para ambiente permanente e Nd o número de animais com registros.

Foram estimadas funções de covariâncias para os componentes genético aditivo e de ambiente permanente, modeladas por meio de polinômios ortogonais, em uma escala de Legendre, de ordens cúbica e quíntica; funções de Wilmink, descritas como: $W(t)=a_{0}+a_{1} t+a_{2} \exp (-b t)$; e funções de Ali e Schaeffer, descritas como: $R(t)=a_{0}+a_{1} u+a_{2} u^{2}+a_{3} v+a_{4} v^{2}$, sendo $u=t / 305 \quad \mathrm{e}$ $v=\ln (305 / t)$. Na função de Wilmink, foram feitas modificações no termo exponencial $a_{2} \exp (-b t)$, em que $b$ foi mantido com o valor de $-0,05$, padrão da função proposta por Wilmink (W05); -0,0565 (W565) obtido pela regressão nãolinear, por meio do procedimento NLIN do SAS (1999); e-0,10 (W10) de acordo com Brotherstone et al. (2000).

As pressuposições em relação aos componentes são:

$$
E\left[\begin{array}{c}
y \\
a \\
a p \\
e
\end{array}\right]=\left[\begin{array}{cl}
X b \\
0 \\
0 \\
0
\end{array}\right] ; \quad \begin{aligned}
& V(a)=K_{A} \otimes A ; \\
& V(a p)=K_{A P} \otimes I_{N d} \\
& \\
& V(e)=R
\end{aligned}
$$

sendo $\mathrm{K}_{\mathrm{A}} \mathrm{e} \mathrm{K}_{\mathrm{AP}}$ as matrizes de (co)variâncias entre os coeficientes de regressão aleatórios genético aditivos e de ambiente permanente, respectivamente; A, a matriz de parentesco; $\mathrm{I}_{\mathrm{Nd}}$, a matriz identidade de dimensão $\mathrm{N}_{\mathrm{d}} ; \otimes$, o produto de Kroenecker; e R, uma matriz diagonal de variâncias residuais.

Primeiramente, assumiu-se variância residual homogênea durante toda a lactação. Depois, considerou-se hetero-

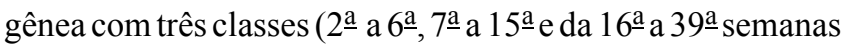
de lactação) e, posteriormente, com quatro classes ( $2 \underline{a}$ a $6 \underline{a}$,

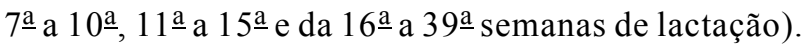

Desse modo, foram comparados os seguintes modelos: polinômio ortogonal de Legendre de ordens cúbica e quíntica, assumindo variância residual heterogênea; função de Ali e Schaeffer, com a variância residual homogênea e heterogênea; e função de Wilmink com modificações no termo exponencial e variância residual homogênea e heterogênea.

O número de parâmetros estimados para cada modelo foi de $\frac{k(k+1)}{2}$ coeficientes de regressão para os efeitos genéticos aditivos e de ambiente permanente, além das classes da variância residual, em que $k$ é a ordem de ajuste das funções paramétricas ou do polinômio ortogonal de Legendre.

A estimativa de herdabilidade para cada dia $t$ de controle foi obtida da seguinte forma: $h_{t_{i}}^{2}=\frac{\hat{g}_{t_{i}}}{\hat{g}_{t_{i}}+\hat{p}_{t_{i}}+\hat{\sigma}_{e}^{2}}$ e as estimativas de correlações genéticas e de ambiente permanente, entre as medidas nos diferentes controles, $t_{i}$ e $t_{j}$ foram definidas respectivamente, por: $r_{g t_{i} t_{j}}=\frac{\hat{g}_{t_{i} t_{j}}}{\sqrt{\left(\hat{g}_{t_{i} t_{j}} * \hat{g}_{t_{i} t_{j}}\right)}} \mathrm{e}$ $r_{\hat{p} t_{i} t_{j}}=\frac{\hat{p}_{t_{i} t_{j}}}{\sqrt{\left(\hat{p}_{t_{i} t_{j}} * \hat{p}_{\left.t_{i} t_{j}\right)}\right)}}$. As estimativas de (co)variância genética e de ambiente permanente para o dia $t$ foram descritas como: $\hat{g}_{t_{i} t_{j}}=Z_{t_{i}}^{\prime} k_{a} Z_{t_{j}}$ e $\hat{p}_{t_{i} t_{j}}=Z_{t_{i}}^{\prime} k_{p} Z_{t_{j}}$, em que $i=2,3, \ldots . .39 e j=2,3, \ldots ., 39$. Os componentes de (co)variância foram estimados pelo método da máxima verossimilhança restrita (REML), utilizando-se a opção DXMRR do pacote estatístico DFREML (Meyer, 1998). A comparação entre os modelos foi feita pelo Critério de Informação de Akaike (AIC) e o critério de informação Bayesiano de Schwar(BIC), que permitem comparação entre modelos não-aninhados. Para AIC e BIC, o valor para comparação é obtido conforme a seguir:

$$
\begin{gathered}
A I C=-2 \ln L+2 p \\
B I C=-2 \ln L+p \ln (N-r)
\end{gathered}
$$

em que: $p$ refere-se ao número de parâmetros do modelo; $\mathrm{N}$, ao número total de observações; e r, ao posto da matriz $\mathrm{X}$, matriz de incidência para os efeitos fixos. Menores valores de AIC e BIC indicam modelos mais adequados. Além disso, as estimativas de variâncias genética e fenotípica e as herdabilidades obtidas pelos modelos mais adequados, de acordo com AIC e BIC, foram comparadas com as análises univariadas para a produção de leite no dia do controle realizadas por Sarmento et al. (2005). 


\section{Resultados e Discussão}

O resumo das análises com $\mathrm{Ln}$ da função de verossimilhança (Ln L) e dos critérios de informação de Akaike (AIC) e Bayesiano (BIC) é apresentado na Tabela 1.

Ao analisar os diferentes expoentes para a função de Wilmink e considerar a variância residual homogênea, observou-se que o modelo W05 apresentou os melhores valores de AIC, BIC e Ln L. Assumindo-se a variância residual heterogênea, o modelo W05 com quatro classes residuais foi o mais adequado para o ajuste dos dados. $\mathrm{O}$ mesmo foi observado para os modelos empregando a função de Ali e Schaeffer (quatro classes residuais). El Faro \& Albuquerque (2003), ao trabalharem com primeiras lactações de vacas da raça Caracu, relataram que o modelo com homogeneidade de variâncias residuais foi inadequado.

Entre as funções paramétricas, a de Ali e Schaeffer foi a mais adequada, mas apresentou problemas quanto à convergência, em virtude do maior número de parâmetros a serem estimados, como observado por Jamrozik et al. (1997). Este problema não ocorreu com o polinômio de Legendre, que tem o mesmo número de parâmetros da função de Ali e Schaeffer. Além disso, o modelo LEG-5 $(\mathrm{e}=4)$, seguido do $\mathrm{AS}(\mathrm{e}=4)$, foi o que apresentou os melhores valores para AIC e Ln L. Contudo, pelo BIC, o modelo LEG-5

Tabela 1 - Número de classes para variâncias residuais (e), número de parâmetros estimados (p), critério de informação de Akaike (AIC), critério de informação Bayesiano (BIC) e função de verossimilhança (Log $L$ ) para os diferentes modelos

Table 1 - Number of residual classes (e), number of estimated parameters (p), Akaike information criterion (AIC), Bayesian information criterion (BIC) and likelihood function ( $L n L)$ for different models

\begin{tabular}{|c|c|c|c|c|c|}
\hline Modelo & $\mathrm{e}$ & $\mathrm{P}$ & AIC & BIC & Ln L \\
\hline \multicolumn{6}{|c|}{ Wilmink } \\
\hline W $05^{1}$ & 1 & 13 & -3905 & -3813 & 1966 \\
\hline W 05 & 3 & 15 & -4003 & -3896 & 2016 \\
\hline W 05 & 4 & 16 & -4073 & -3958 & 2052 \\
\hline $\mathrm{W} 10^{2}$ & 1 & 13 & -3660 & -3567 & 1843 \\
\hline W $565^{3}$ & 1 & 13 & -3861 & -3768 & 1943 \\
\hline \multicolumn{6}{|c|}{ Ali e Schaeffer } \\
\hline $\mathrm{AS}^{4}$ & 1 & 31 & -4568 & -4346 & 2315 \\
\hline AS & 3 & 33 & -4634 & -4399 & 2350 \\
\hline AS & 4 & 34 & -4694 & -4451 & 2381 \\
\hline \multicolumn{6}{|c|}{ Legendre } \\
\hline LEG $-3^{5}$ & 3 & 15 & -4201 & -4093 & 2115 \\
\hline LEG-3 & 4 & 16 & -4267 & -4152 & 2149 \\
\hline LEG-5 ${ }^{6}$ & 3 & 33 & -4689 & -4453 & 2378 \\
\hline LEG-5 & 4 & 34 & -4756 & -4513 & 2412 \\
\hline
\end{tabular}

$1,2,3$ = função de Wilmink com expoentes iguais a -0,05;-0,10; e -0,0565, respectivamente; 4 = função de Ali e Schaeffer; $5,6=$ polinômios de Legendre de ordem cúbica e quíntica, respectivamente.

$1,2,3=$ Wilmink function with exponents equal to $-0.05 ; 0.10 ; \mathrm{e}-0.0565$, respectively; $4=\mathrm{Ali}$ \& Schaeffer function; 5,6 = cubic and quintic order Legendre orthogonal polynomial.
( $\mathrm{e}=4$ ), seguido do LEG-5 ( $\mathrm{e}=3$ ), foi o que proporcionou o melhor ajuste dos dados. Vale ressaltar que ambos, AIC e BIC, penalizam modelos com maior número de parâmetros. Entretanto, para BIC, esta penalidade é mais rigorosa, tendendo a favorecer modelos mais parcimoniosos (NunezAntón \& Zimmerman, 2000).

Na Figura 2 são apresentadas as variâncias fenotípicas, de ambiente permanente e genéticas, estimadas pelos modelos que melhor descreveram os dados, de acordo com os critérios adotados, juntamente com as estimativas obtidas pelas análises unicaracterísticas realizadas por Sarmento et al. (2005). As estimativas de variância fenotípica (Figura 2a) obtidas pelos modelos que empregaram o polinômio ortogonal de Legendre de ordem quíntica (LEG-5), com três $(\mathrm{e}=3)$ e quatro $(\mathrm{e}=4)$ classes de variâncias residuais, foram praticamente as mesmas. No modelo que usou a função de Ali e Schaeffer (AS) com quatro classes residuais $(e=4)$, observaram-se pequenas diferenças nas estimativas de

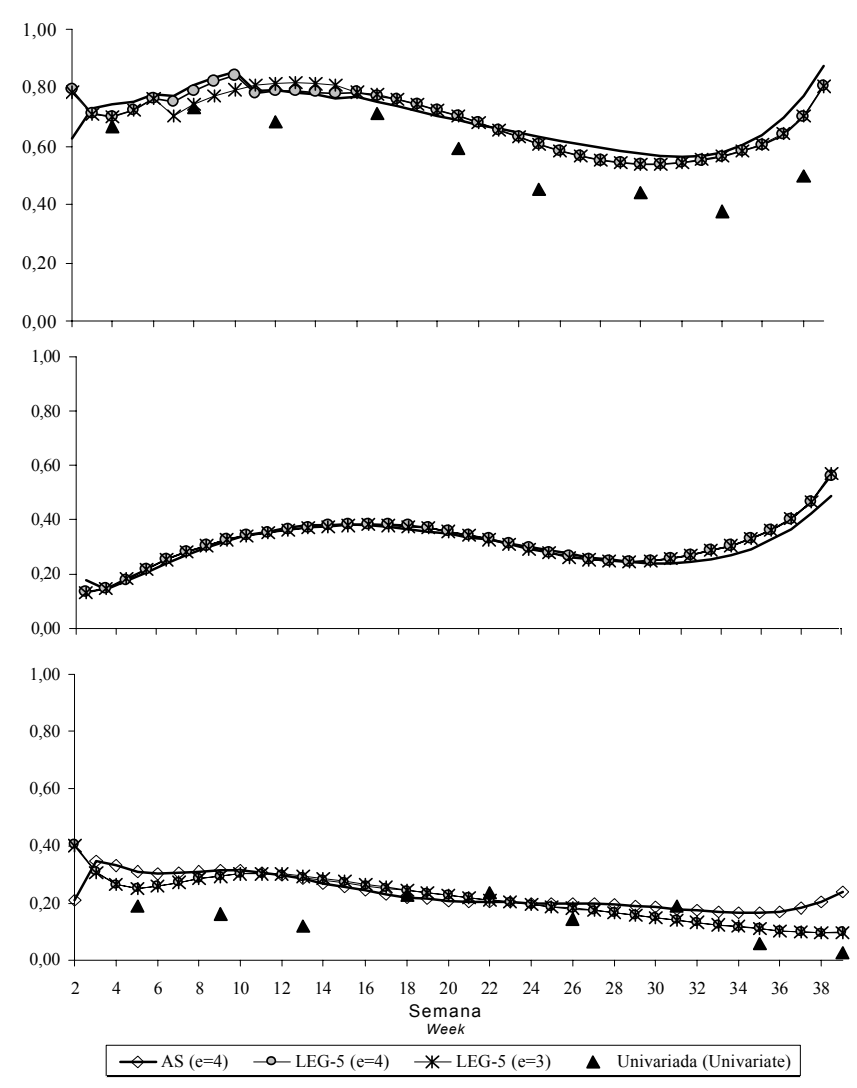

Figura 2 - Estimativas de variâncias fenotípicas (a), de ambiente permanente (b) e genéticas (c) obtidas utilizando-se a função Ali e Schaeffer (AS), com quatro classes residuais $(e=4)$ e o Polinômio de Legendre de ordem quíntica LEG-5, com três $(e=3)$ e quatro $(e=4)$ classes residuais.

Figure 2 - Phenotypic (a), permanent environmental (b) and genetic (c) variance estimates obtained using Ali e Schaeffer (AS) function with four residual classes $(e=4)$ and fifth order Legendre polynomial (LEG-5) with three $(e=3)$ and four $(e=4)$ residual classes. 
variância fenotípica em relação às obtidas pelo LEG-5 $(\mathrm{e}=3)$ e LEG-5 ( $\mathrm{e}=4)$ no início e no final da lactação. As estimativas mostraram tendência de aumento até a $10^{\mathrm{a}}$ semana $(0,84)$, exceto da $6 \underline{\text { a }}$ para $7 \underline{\text { a }}$, em que houve redução da variância fenotípica para o LEG-5 (e=3). Este período é caracterizado pela mudança da primeira para a segunda classe residual. Além disso, coincide com o pico de lactação, característico de raças especializadas, em torno de 60 dias após o parto. Após a $10 \underline{a}$ semana, estas estimativas diminuíram até a 32a semana $(0,54)$, voltando a aumentar no final da lactação e alcançando valores próximos a 0,87 na 39 a semana. Possivelmente, este aumento no final da lactação seja decorrente do menor número de informações disponíveis neste período.

Quanto às variâncias de ambiente permanente estimadas (Figura 2b), os modelos tiveram comportamentos semelhantes, com estimativas mais baixas no início da lactação $(0,13)$, inferiores às observadas para estimativas de variância genética aditiva (Figura $2 \mathrm{c}$ ). Após a $7 \underline{\mathrm{a}}$ semana $(0,28)$, as variâncias resultantes do efeito de ambiente permanente tenderam a aumentar até a $17 \underline{\text { a }}(0,37)$, decrescendo em seguida até a $30^{\mathrm{a}}(0,25)$ e aumentando até o final da lactação $(0,50)$. Neste período, as variâncias de ambiente permanente foram superiores às genéticas.

As estimativas de variâncias genéticas (Figura 2c) obtidas pelos modelos LEG-5 ( $\mathrm{e}=3$ ), LEG-5 ( $\mathrm{e}=4)$ e AS ( $\mathrm{e}=4$ ) foram praticamente as mesmas, exceto no início e no final da lactação, em que $\operatorname{AS}(\mathrm{e}=4)$ apresentou, respectivamente,

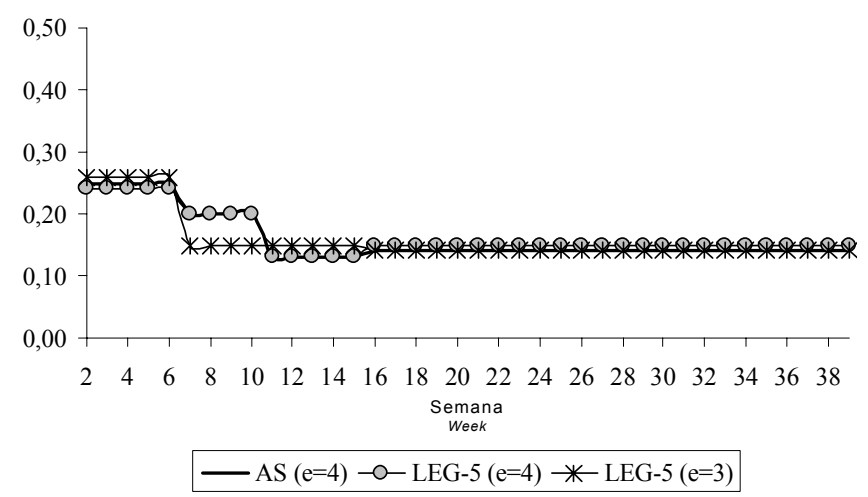

Figura 3 - Estimativas de variância residual obtidas com a função Ali \& Schaeffer (AS), o polinômio de Legendre, de ordem quíntica (LEG-5), com quatro classes residuais $(e=4)$, definidas pelos intervalos da $2 \underline{a}$ a $6 \underline{a}$; $7 \underline{a}$ a $10 \underline{a}$; $11 \underline{\text { a }}$ a 15무 e da 16a a 39a semanas de lactação e o polinômio de Legendre, de ordem quíntica (LEG-5) com três classes residuais $(\mathrm{e}=3)$, definidas pelos intervalos da $2 \underline{\mathrm{a}}$ a $6 \underline{\mathrm{a}} ; \mathbf{7} \underline{\mathrm{a}}$ a 15á ; e da 16a a 39a semanas de lactação.

Figure 3 - Residual variance estimated obtaines using Ali e Schaeffer function (AS) and fifth order Legendre polynomial (LEG-5) with four residual classes $(e=4)$ defined by $2^{\text {nd }}$ to $6^{\text {th }} ; 7^{\text {th }}$ to $10^{\text {th }} ; 11^{\text {st }}$ to $15^{\text {th }}$ and $16^{\text {th }}$ to $39^{\text {th }}$ weeks of lactation and fifth order Legendre polynomial (LEG-5) with three residual classes $(e=3)$ defined by $2^{\text {nd }}$ to $6^{\text {th }}$; $7^{\text {th }}$ to $15^{\text {th }}$; and $16^{\text {th }}$ to $39^{\text {th }}$ weeks of lactation. estimativas inferiores $(0,21)$ e superiores $(0,23)$ às obtidas pelos modelos LEG-5 (e=3) e LEG-5 ( $\mathrm{e}=4$ ). De modo geral, as estimativas de variância genética decresceram ao longo da lactação, o que difere dos resultados encontrados por Sarmento et al. (2003), que trabalharam com polinômios de Legendre de 5 a ordem em cabras mestiças Pardo-Alpina x Gurguéia e verificaram aumento da variância genética aditiva no decorrer da lactação. Também foi observado que o comportamento das estimativas de variâncias fenotípica (Figura 2a) e genética (Figura 2c) foi semelhante ao encontrado na análise univariada, com modelos de produção no dia do controle, ao se utilizar o mesmo arquivo de dados (Sarmento et al., 2005).

Na Figura 3 são apresentadas as estimativas de variância residual para as classes de lactações. Observou-se que o valor $(0,25)$ obtido para primeira classe foi superior aos demais para os três modelos. Jensen (2001), ao estudar a curva de lactação de bovinos da raça Jersey, encontrou maiores variâncias residuais nas primeiras semanas de lactação, evidenciando a maior dificuldade de controlar as influências ambientais nas primeiras semanas de lactação. A estimativa de variância residual para a primeira classe foi superior às de variância de ambiente permanente e inferior às de variâncias genéticas apenas na $2^{\mathrm{a}}$ semana para $\mathrm{o}$ modelo AS $(\mathrm{e}=3)$. Foram observadas diferenças entre os modelos no período da 7 e $10^{\underline{a}}$ semanas de lactação, obtendo-se menores estimativas pelo LEG-5 $(\mathrm{e}=3)(0,15)$. Após a $10^{\mathrm{a}}$ semana, as estimativas da variância residual $(0,14)$ obtidas pelos três modelos não diferiram.

As estimativas de herdabilidade (Figura 4) obtidas pelos três modelos decresceram ao longo da lactação. Os modelos que empregaram o LEG-5 ( $\mathrm{e}=3)$ e LEG-5 $(\mathrm{e}=4)$ foram bastante semelhantes. O AS $(e=4)$, por sua vez,

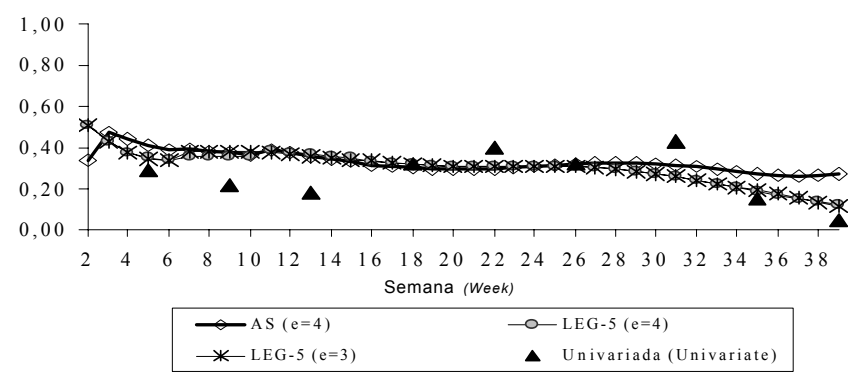

Figura 4 - Herdabilidades para as produções de leite no dia do controle obtidas com a função de Ali e Schaeffer (AS), com quatro classes residuais $(e=4)$, e os polinômios de Legendre de ordem quíntica (LEG-5), com três $(e=3)$ e quatro $(e=4)$ classes residuais.

Figure 4 - Heritability for test day milk yield obtained using Ali and Schaeffer function (AS) with four residual classes $(e=4)$ and fifth order Legendre polynomial (LEG-5) with three $(e=3)$ and four $(e=4)$ residual classes. 
apresentou estimativas divergentes no início e no final da lactação, registrando-se menor valor na 2 a semana e maior da $3 \underline{\text { a }}$ à $6 \underline{\text { a }}$ semana e no final da lactação, após a $27 \underline{\text { a }}$ semana.
As herdabilidades estimadas pelos modelos LEG-5 (e=3) eLEG-5 (e=4) variaram de 0,12 (39-a semana) a 0,51 (2 2 - semana)

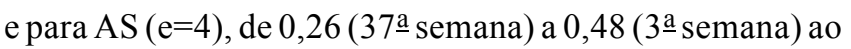
longo da lactação. Os modelos apresentaram estimativas de

Tabela 2 - Estimativas de variâncias (diagonal), covariâncias (abaixo da diagonal) e correlações (acima da diagonal) entre os coeficientes de regressão aleatória, os autovalores $(\lambda)$ e a porção da variância explicada pelo autovalor da matriz de coeficientes

Table 2 - Estimates of variance (diagonal), covariance (below diagonal) and correlation (above diagonal) between random regression coefficients, eigenvalues $(\lambda)$ and portion of the variance explained by eigenvalues of the coefficient matrix

\begin{tabular}{|c|c|c|c|c|c|c|}
\hline 0 & 1 & 2 & 3 & 4 & $\lambda$ & $\begin{array}{c}\% \text { da variância } \\
\text { \% of variance }\end{array}$ \\
\hline \multicolumn{7}{|c|}{ LEG-5 $(\mathrm{e}=3)^{1}$} \\
\hline \multicolumn{7}{|c|}{$\begin{array}{c}\text { Efeito genético aditivo } \\
\text { Addictive genetic effect }\end{array}$} \\
\hline 0,356 & $-0,460$ & $-0,701$ & 0,259 & 0,032 & 0,370 & 85,30 \\
\hline$-0,055$ & 0,040 & $-0,302$ & $-0,326$ & $-0,316$ & 0,036 & 8,30 \\
\hline$-0,033$ & $-0,005$ & 0,006 & $-0,112$ & 0,312 & 0,028 & 6,50 \\
\hline 0,022 & $-0,009$ & $-0,001$ & 0,020 & $-0,794$ & 0,000 & 0,00 \\
\hline 0,001 & $-0,005$ & 0,002 & $-0,008$ & 0,005 & 0,000 & 0,00 \\
\hline \multicolumn{7}{|c|}{$\begin{array}{l}\text { Efeito de ambiente permanente } \\
\text { Permanent environmental effect }\end{array}$} \\
\hline 0,445 & 0,015 & $-0,348$ & 0,430 & 0,003 & 0,458 & 73,30 \\
\hline 0,003 & 0,087 & 0,391 & $-0,287$ & 0,074 & 0,103 & 16,50 \\
\hline$-0,056$ & 0,028 & 0,059 & $-0,193$ & $-0,055$ & 0,043 & 6,90 \\
\hline 0,041 & 0,012 & $-0,007$ & 0,021 & 0,173 & 0,017 & 2,70 \\
\hline 0,001 & 0,002 & $-0,015$ & 0,003 & 0,013 & 0,004 & 0,64 \\
\hline \multicolumn{7}{|c|}{ LEG-5 $(\mathrm{e}=4)^{2}$} \\
\hline \multicolumn{7}{|c|}{$\begin{array}{c}\text { Efeito genético aditivo } \\
\text { Addictive genetic effect }\end{array}$} \\
\hline 0,354 & $-0,461$ & $-0,674$ & 0,251 & 0,053 & 0,367 & 86,14 \\
\hline$-0,055$ & 0,040 & $-0,332$ & $-0,343$ & $-0,268$ & 0,037 & 8,58 \\
\hline$-0,032$ & $-0,005$ & 0,006 & $-0,089$ & 0,264 & 0,022 & 5,10 \\
\hline 0,208 & $-0,009$ & $-0,001$ & 0,019 & $-0,732$ & 0,001 & 0,18 \\
\hline 0,248 & $-0,004$ & $-0,002$ & $-0,008$ & 0,006 & 0,000 & 0,00 \\
\hline \multicolumn{7}{|c|}{$\begin{array}{c}\text { Efeito de ambiente permanente } \\
\text { Permanent environmental effect }\end{array}$} \\
\hline 0,448 & 0,014 & $-0,351$ & 0,424 & 0,000 & 0,460 & 73,38 \\
\hline 0,003 & 0,085 & 0,395 & $-0,284$ & 0,064 & 0,103 & 16,37 \\
\hline$-0,057$ & 0,027 & 0,059 & $-0,189$ & $-0,555$ & 0,043 & 6,81 \\
\hline 0,421 & $-0,012$ & $-0,007$ & 0,022 & 0,144 & 0,017 & 2,71 \\
\hline 0,207 & 0,002 & $-0,015$ & 0,002 & 0,013 & 0,005 & 0,73 \\
\hline \multicolumn{7}{|c|}{$\operatorname{AS}(e=4)^{3}$} \\
\hline \multicolumn{7}{|c|}{$\begin{array}{c}\text { Efeito genético aditivo } \\
\text { Addictive genetic effect }\end{array}$} \\
\hline 170,140 & $-0,998$ & 0,987 & $-0,997$ & 0,990 & 966,469 & 99,70 \\
\hline$-396,019$ & 589,136 & $-0,995$ & 0,994 & $-0,985$ & 2,657 & 0,27 \\
\hline 164,836 & $-309,007$ & 163,804 & $-0,979$ & 0,965 & 0,160 & 0,02 \\
\hline$-87,695$ & 162,666 & $-84,517$ & 45,465 & $-0,998$ & 0,062 & 0,01 \\
\hline 11,583 & $-21,440$ & 11,078 & $-6,033$ & 0,804 & 0,000 & 0,00 \\
\hline \multicolumn{7}{|c|}{$\begin{array}{c}\text { Efeito de ambiente permanente } \\
\text { Permanent environmental effect }\end{array}$} \\
\hline 53,479 & $-0,962$ & 0,716 & $-0,992$ & 0,978 & 244,472 & 0,9374 \\
\hline$-85,818$ & 148,792 & $-0,874$ & 0,925 & $-0,888$ & 16,020 & 0,0614 \\
\hline 34,928 & $-71,947$ & 44,497 & $-0,638$ & 0,574 & 0,280 & 0,0011 \\
\hline$-26,929$ & 41,881 & $-15,803$ & 13,772 & $-0,996$ & 0,032 & 0,0001 \\
\hline 3,668 & $-5,559$ & 1,964 & $-1,896$ & 0,263 & 0,000 & 0,0000 \\
\hline
\end{tabular}

1, 2 Polinômio de Legendre de ordem quíntica com três e quatro classes residuais, respectivamente; ${ }^{3}$ Função de Ali e Schaeffer com quatro classes residuais.

1,2 Quintic order Legendre orthogonal polynomial with three and four residual classes, respectively; ${ }^{3}$ Ali e Schaefferfunction with four residual classes. 
herdabilidades semelhantes às obtidas pela análise univariada, com modelos de produção no dia do controle. Os estudos com caprinos encontrados na literatura relatam herdabilidades de 0,12 a 0,30 (Breznik et al., 2000; Gonçalves et al., 2001; Oliveira et al., 2003; Lôbo et al., 2003), mas envolvem apenas a produção no dia do controle, por meio de um modelo de repetibilidade, ou a produção total, por meio dos modelos tradicionais.

Constam na Tabela 2 as estimativas de (co)variâncias e correlações entre os coeficientes de regressão para os efeitos genético aditivo e de ambiente permanente para os três modelos. As correlações entre os regressores para o modelo $\mathrm{AS}(\mathrm{e}=4)$ foram próximas à unidade e provavelmente são a causa das dificuldades encontradas para a convergência no processo iterativo. As correlações entre os regressores para os efeitos genético aditivo e de ambiente permanente, peloLEG-5 (e=3), variaram de-0,79a 0,31 e de-0,34 a 0,43 e, pelo LEG-5 (e=4), de-0,73 a 0,26 e de-0,55 a 0,42, respectivamente.

Na proporção da variância explicada pelos autovalores, observou-se, pelos três modelos, que o primeiro e o segundo autovalores explicaram a maior parte da variância, indicando que um modelo quadrático poderia ser adequado para ajustar a variância nos dados. Contudo, esse modelo não foi indicado pelo AIC e BIC (Tabela 1). De acordo com Legarra et al. (2004), a redução de dimensionalidade decorrente da eliminação de autovalores $(0,00)$ não é indicada em todos os casos, podendo resultar em ajuste inadequado no início e no final da trajetória. No modelo quadrático, as estimativas de herdabilidades variaram de 0,07 a 0,25, de modo que os valores mais baixos foram obtidos no final da lactação. Essas estimativas estão de acordo com os valores de herdabilidade relatados em outros trabalhos, com

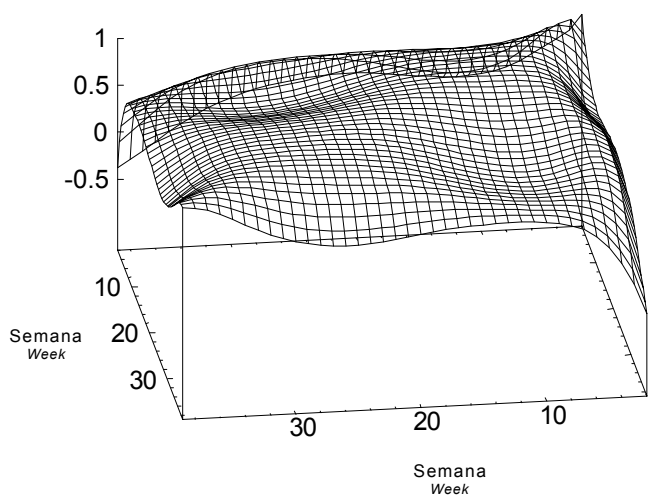

Figura 5 - Correlações genéticas estimadas entre as produções de leite semanais, de acordo com o modelo que usou a função de Ali e Schaeffer (AS) com quatro classes residuais $(e=4)$.

Figure 5 - Genetic correlation estimated between for day week milk yield obtained using Ali and Schaeffer function (AS) with four residual classes $(e=4)$.
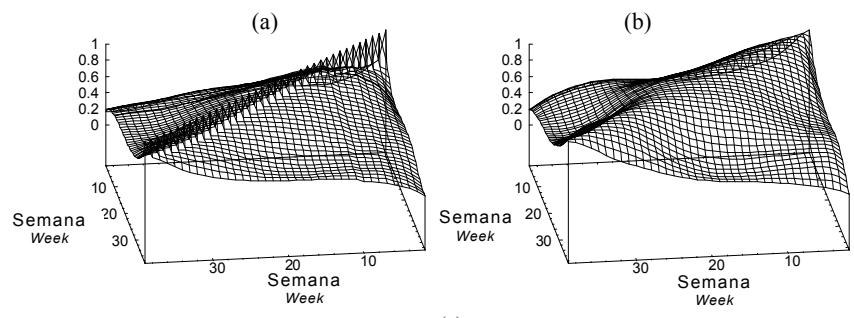

(c)

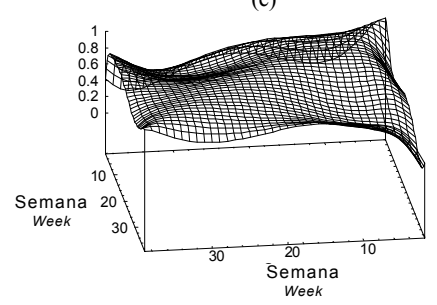

Figura 6 - Correlações fenotípicas (a), de ambiente permanente (b) e genéticas (c) estimadas entre as produções de leite semanais, de acordo com o modelo que usou os polinômios de Legendre de ordem quíntica LEG-5 com quatro classes residuais $(e=4)$.

Figure 6 - Phenotypic (a), permanent environmental (b) and genetic (c) correlation estimated between weekly milk yield obtained using fifth order Legendre polynomial (LEG-5) with four residual classes $(e=4)$.

caprinos, utilizando modelos tradicionais. Entretanto, em relação às obtidas pelo $\operatorname{AS}(\mathrm{e}=4)(0,26 \mathrm{a} 0,48), \mathrm{LEG}-5(\mathrm{e}=3)$ e LEG-5 $(\mathrm{e}=4)(0,12$ a 0,51$)$ estas foram bem menores. Além disso, estudos realizados com regressão aleatória em bovinos de leite (Cobuci, 2002; Freitas, 2003; Araújo, 2003) têm relatado estimativas de herdabilidade superiores às obtidas pelos modelos tradicionais, o que não foi verificado com o uso do modelo quadrático.

As estimativas de correlações genéticas obtidas pelos três modelos não foram sempre menores quanto mais distantes os controles leiteiros (Figuras 5 e 6c), como seria esperado. Tenderam a diminuir quanto mais distantes os controles leiteiros, contudo, em alguns pontos esse comportamento não ocorreu. Além disso, o modelo com a função de Ali e Schaeffer (Figura 5) resultou em estimativas que variaram de $-0,052$ a 0,99 entre os diferentes controles, sendo as negativas obtidas entre os controles mais distantes (início e final da lactação). Resultados semelhantes foram encontrados por Costa et al. (2002) e Freitas et al. (2004). De acordo com Brotherstone et al. (2000), funções paramétricas, como a de Ali e Schaeffer, apresentam inabilidade em modelar a associação entre o início e o final da lactação, resultando em correlações negativas entre estes períodos.

As correlações fenotípicas, de ambiente permanente e genéticas (Figura 6) estimadas pelos modelos usando LEG-5 $(\mathrm{e}=3)$ e LEG-5 $(\mathrm{e}=4)$ foram semelhantes e, por isso, foram 
apresentadas apenas para o LEG-5 (e=4), também apontado como o mais adequado pelo AIC e BIC (Tabela 1). As estimativas de correlações fenotípicas (Figura 6a) foram menores que as genéticas e de ambiente permanente, variando de 0,17 a 0,84 . Resultados semelhantes também foram encontrados por El Faro \& Albuquerque (2003), em bovinos da raça Caracu. As correlações de ambiente permanente variaram de 0,18 a 0,99 . As correlações genéticas (Figura 6c) foram todas positivas, variando de 0,42 a 1,00 entre diferentes controles. Contudo, como mencionado, as correlações oscilaram, não sendo sempre menores quanto mais distantes os controles leiteiros. Meyer (1998), ao trabalhar com a característica peso, em bovinos de corte, relatou que, ao aumentar a ordem de ajuste (de 4 para 6), as estimativas de correlações flutuaram consideravelmente, especialmente na extremidade da superfície. Outro fato que também pode ter influenciado essas estimativas é o pequeno volume de dados em análise.

\section{Conclusões}

O polinômio de Legendre de ordem quíntica, com quatro e três classes residuais, e a função de Ali e Schaeffer, com quatro classes residuais, modelaram adequadamente a variância fenotípica, diferindo na partição para as variâncias de ambiente permanente, genética e residual apenas no início e no final da lactação.

O polinômio de Legendre de ordem quíntica mostrou-se mais adequado que a função de Ali e Schaeffer para os estudos genéticos da produção de leite no dia do controle de cabras da raça Alpina.

\section{Literatura Citada}

ALI, E.; SCHAEFFER, L.R. Accounting for covariances among test day milk yields in dairy cows. Canadian Journal Animal Science, v.67, n.3, p.637-644, 1987.

ARAÚJO, C.V. Modelos de regressão aleatória para a avaliação genética da produção de leite na raça Holandesa. Viçosa: Universidade Federal de Viçosa, 2003, 78p. Tese (Doutorado em Zootecnia) - Universidade Federal de Viçosa, 2003.

BREZNIK, S.; MALOVRH, S.; KOVAC, M. et al. Additive genetic and environmental variance components for milk traits in goat with test day model. Zootehnika, v.76, n.1, p.61-66, 2000.

BROTHERSTONE, S.; WHITE, L.M.S.; MEYER, K. Genetic modelling of daily milk yield using orthogonal and parametric curves. Animal Science, v.70, p.407-415, 2000.

COBUCI, J.A. Uso de modelos de regressão aleatória na avaliação da persistência na lactação de animais da raça Holandesa. Viçosa: Universidade Federal de Viçosa, 2002, 78p. Tese (Doutorado em Zootecnia) - Universidade Federal de Viçosa, MG, 2002.

COSTA, C.N.; MELO, C.M.R.; MACHADO, C.H.C. et al. Avaliação de funções polinomiais para ajuste da produção de leite no dia do controle de primeiras lactações de vacas Gir com modelo de regressão aleatória. In: REUNIÃO ANUAL DA SOCIEDADE
BRASILEIRA DE ZOOTECNIA， 39., 2002, Recife, Pernambuco. Anais... Recife: SBZ, 2002. CD-ROM. Melhoramento Animal. 04sbz499.pdf.

EL FARO, L.; ALBUQUERQUE, L.G. Utilização de modelos de regressão aleatória para a produção de leite no dia do controle, com diferentes estruturas de variâncias residuais. Revista Brasileira de Zootecnia, v.32, n.5, p.1104-1113, 2003.

FREITAS, M.S. Utilização de modelos de regressão aleatória na avaliação genética de animais da raça girolando. Viçosa: Universidade Federal de Viçosa, 2003. 78p. Dissertação (Mestrado em Zootecnia) - Universidade Federal de Viçosa, MG, 2003.

FREITAS, M.S.; REIS FILHO, J.C.; FREITAS, A.S. et al. Estimativas de parâmetros genéticos da produção de leite no dia do controle de vacas Girolando utilizando a função de Ali e Schaeffer em modelo de regressão aleatória. REUNIÃO ANUAL DA SOCIEDADE BRASILEIRA DE ZOOTECNIA, 41., 2004, Campo Grande. Anais...Campo Grande: SBZ, 2004. CD-ROM. Melhoramento Animal. MELH136.pdf.

GONÇALVES, H.C.; SILVA, A.M.; WECHSLER, F.S.; et al. Fatores genéticos e de meio na produção de leite de caprinos leiteiros. Revista Brasileira de Zootecnia, v.30, n.3, p.719-729, 2001.

GONÇALVES, H.C.; SILVA, M.A.; WECHSLER, F.S. et al. Parâmetros e tendência genética da produção de leite de cabra no Brasil. Revista Brasileira de Zootecnia, v.31, n.6, p.2204-2208, 2002.

JAKOBSEN, J.H.; MADSEN, P.; JENSEN, J. et al. Genetic parameters for milk production and persistency for Danish Holsteins estimated in random regression models using REML. Journal of Dairy Science, v.85, n.6, p.1607-1615, 2002.

JAMROZIK, J.; SCHAEFFER, L.R.; DEKKERS, J.C.M. Genetic evaluation of dairy cattle using test day yields and random regression model. Journal of Dairy Science, v.80, n.6, p.12171226, 1997.

JENSEN, J. Genetic evaluation of dairy cattle using test-day models. American Dairy Science Association, v.84, n.12, p.2803$2812,2001$.

LÔBO, R.N.B.; SILVA, F.L.R. Herdabilidade para produção de leite em cabras das raças Saanen e Anglo Nubiana. In: REUNIÃO ANUAL DA SOCIEDADE BRASILEIRA DE ZOOTECNIA, 40., 2003, Santa Maria. Anais... Santa Maria: SBZ, 2003. CDROM. Melhoramento Animal. 4_10.pdf.

LEGARRA, A.; MISZTAl, I.; BERTRAND, J.K. Constructing covariance functions for random regression models for growth in Gelbvieh beef cattle. Journal of Animal Science, v. 82, p.1564-1571, 2004.

MEYER, K. DXMRR - A program to estimate covariance functions for longitudinal data by REML. In: 6th. WORLD CONGRESS OF GENETICS APPLIED TO LIVESTOCK PRODUCTION, 6., 1998, Armidale. Proceedings... Armidale: University of New England, 1998. CD-ROM.

MEYER, K. Random regression models for analyses of longitudinal data in animal breeding. In: SESSION OF THE INTERNATIONAL STATISTICAL InSTITUTE, 54., 2003, Berlim. Proceedings... Berlim, 2003.

NUNEZ-ANTÓN, V.N.; ZIMMERMAN, D.L. Modelling nonstationary longitudinal data. Biometrics, v.56, p.699-705, 2000.

OLIVEIRA, S.M.P.; LIMA, A.C.W.; LIMA, F.A.M. Estimativas de herdabilidade e correlações genéticas de produções parciais e total de leite de cabras mestiças. In: REUNIÃO ANUAL DA SOCIEDADE BRASILEIRA DE ZOOTECNIA, 40., 2003, Santa Maria. Anais... Santa Maria: SBZ, 2003. CD-ROM. Melhoramento Animal. 4_74.pdf.

PIMENTA FILHO, E.C.; SARMENTO, J.L.R.; RIBEIRO, M.N. Efeitos genéticos e ambientais que afetam a produção de leite e duração da lactação de cabras, estiças no estado da Paraíba. Revista Brasileira de Zootecnia, v.31, n.6, p.1426-1431, 2004.

POLL, M.H.; MEUWISSEN, T.H.E. Prediction of daily milk yields from a limited number of test days using test day models. Journal of Dairy Science, v. 82, n.7, p.1555-1564, 1999

SARMENTO, J.L.R.; TORRES, R.A.; BREDA, F.C. et al. Parâmetros genéticos para a produção de leite no dia do controle de cabras 
mestiças por meio de regressão aleatória. In: REUNIÃO ANUAL DA SOCIEDADE BRASILEIRA DE ZOOTECNIA, 40., 2003, Santa Maria. Anais... Santa Maria: SBZ, 2003. CD-ROM. Melhoramento Animal.4 121.pdf.

SARMENTO, J.L.R.; REIS FILHO, J.C.; ALBUQUERQUE, L.G. et al. Avaliação genética de caprinos por meio da produção de leite no dia de controle. Revista Brasileira de Zootecnia, 2005. (enviado para publicação)

STATISTICAL ANALYSES SYSTEM - SAS. SAS/STAT user'guide, version 8.0 ed. Cary: 1999. v.1, 943p.

SCHAEFFER, L.R. Application of regression models models in animal breeding. Livestock Production Science, v.86, p. 35-45, 2004.

SOARES FILHO, G.; MCMANUS, C.; MARIANTE, A.S. Fatores genéticos e ambientais que influenciam algumas características de reprodução e produção de leite em cabras no Distrito Federal. Revista Brasileira de Zootecnia, v.30, n.1, p.53-59, 2001.
SULLIVAN, B.; WIGGANS, G. Genetic evaluation of dairy goats in the United States and Canada. In: INTERNATIONAL CONFERENCE ON GOATS, 7., 2000, Poities. Proceedings... Poities, 2000, CD-ROM.

WILMINK, J.B.M. Efficiency of selection for different cumulative milk, fat and protein yields in first lactation. Livestock Production Science, v.17, n.3, p.211-224, 1987. 